\title{
Reliability optimization of complex systems using sharp lower bounds
}

\author{
M. SOUISSI ${ }^{1}$ and Y. SMEERS \\ CORE, Université Catholique de Louvain \\ 34, voie du roman pays, 1348 Louvain-la-Neuve, Belgium. \\ Fax: (32)10-474301, e-mail: souissi@core.ucl.ac.be
}

\begin{abstract}
Reliability of production systems has become a prominent issue especially after the introduction of process oriented manufacturing techniques, (ex: Just-in-Time, M.R.P., O.P.T.). In general the reliable performance of a system is of the utmost importance in many other industrial, security and everyday life situations as well. This paper deals with reliability optimization of coherent systems by using redundancy. An algorithm based on some lower bounds of system reliability is presented and the numerical results are compared to the so far existing results.
\end{abstract}

Keywords

Reliability, optimization, nonlinear programming.

\section{INTRODUCTION}

The reliable performance of a system for a mission under various conditions is of the utmost importance in many industrial, security and everyday life situations. The increasing need for highly reliable systems and components of greater safety and less cost, has given rise to several developments in the past three decades. A good deal of effort has been centered in the field of optimal redundancy allocation. Most of the papers have considered the case of $n$-stage series models or series-parallel- series models, and developed different techniques to optimize their redundancy (dynamic programming, Linear integer programming, nonlinear programming with integer variables, geometric programming, ...). Some authors have given heuristics to optimize the reliability in complex systems. This paper deals with optimization of components redundancy into a coherent system. The algorithm can be used by a system designer faced with the problem of maximizing system reliability $R(S)$, subject to constraints (possibly nonlinear) on system resourses such as cost, weight, and power.

This problem was deeply studied in the case of series systems; the reader may refer to Bellman et al. (1958) and Tillman et al. (1977) In the case of complex systems, some

\footnotetext{
${ }^{1}$ Corresponding author: on leave from Ecole Mohammadia d'Ingénieurs, Rabat, Maroc
} 
Heuristics have been developed, see Aggarwal (1976), Misra et al. (1979), Shi (1987), Yasutake-Baker et al. (1985).

The work presented here is based on the equivalent representation of coherent systems in terms of its minimal cuts or minimal paths; see Barlow and Prochan (1975). In this fashion we have a lower bound on system reliability which will be maximized instead of $R(S)$.

Before introducing our algorithm, here are some fundamental definitions as introduced by Barlow et al. (1975).

Coherent system: A system $S$ of components is coherent if: (a) its structure function $\Phi$ is increasing, and (b) each component is relevant.

path : is a vector $x=\left(x_{1}, x_{2}, \ldots, x_{n}\right)$ such that $\Phi(x)=1$

cut : is a vector $x=\left(x_{1}, x_{2}, \ldots, x_{n}\right)$ such that $\Phi(x)=0$

minimal path : is a path $x$ such that $\forall y<x, \Phi(y)=0$. Let $P=\left\{i: x_{i}=1\right\}, P$ is called a minimal path set. It is a minimal set of components whose functioning insure the functioning of the system. For each minimal path set $P_{j}$ we assign a series structure $\rho_{j}(x)=\prod_{i \in P_{j}} x_{i}$.

minimal cut : is a cut $x$ such that $\forall y>x, \Phi(y)=1$. Let $K=\left\{i: x_{i}=0\right\} \quad K$ is called a minimal cut set. It is a minimal set of components whose failure causes the system to fail. For each minimal cut set $K_{j}$ we assign a parallel structure $\kappa_{j}(x)=\coprod_{i \in K_{j}} x_{i}$.

Proposition 1 A coherent system $(S, \Phi)$ functions if and only if at least one of the minimal path structures is functioning. That is $\Phi(X)=\coprod_{j=1}^{p} \rho_{j}(x)=\coprod_{j=1}^{p} \prod_{j \in P_{j}} x_{i}$ where $p$ is the number of minimal path sets.

Similarly, $(S, \Phi)$ fails if and only if at least one of the minimal cut structures fails. That is $\Phi(X)=\prod_{j=1}^{k} \kappa_{j}(x)=\prod_{j=1}^{k} \prod_{j \in K_{j}} x_{i}$ where $k$ is the number of minimal cut sets.

\section{ALGORITHM BASED ON MINIMAL CUT SETS}

The main assumptions considered here are the statistical independency between the component states and the separability of constraints in terms of variables $m_{j}$. The problem of constrained redundancy optimization can be formulated as follows:

Maximize $R(S)$

s.t. $\quad \sum_{j=1}^{N} g_{i j}\left(m_{j}\right) \leq b_{i} \quad i=1,2, \ldots, r$

and $\quad m_{j} \in \mathbb{N}^{*} \quad j=1, \ldots, N$

In general, it is quite difficult to compute the exact expression of the reliability function for complex systems. Ball (1979) has shown that all nontrivial reliability problems of general systems are $N P$ - hard. Even for small systems, the mathematical expression of reliability function, as we will see in example 1 , can be very complicated and heavy to 
manipulate. We have thus chosen to use an approach by lower bounds. In the first part, we consider the lower bound of system reliability. It is obtained from the representation of the initial system $S$ in terms of its minimal cut sets given by $\underline{R}(S)=\prod_{l=1}^{k} R_{l}(S)$, where $R_{l}(S)$ is the reliability of the $l^{\text {th }}$ minimal cut. Instead of maximizing $R(S)$, we maximize $\underline{R}(S)$. The resulting problem is:

Maximize $\quad \underline{R}(S)=\prod_{l=1}^{k}\left(1-\prod_{j \in K_{l}}\left(1-p_{j}\right)^{m_{j}}\right)$

s.t. $\quad \sum_{j=1}^{N} g_{i j}\left(m_{j}\right) \leq b_{i} \quad i=1,2, \ldots, r$

and $\quad m_{j} \in \mathbb{N}^{*} \quad j=1, \ldots, N$

To solve this problem we need the following lemma:

Lemma 1 Let $f$ be a positive function defined on $D$ and $g$ be a function defined by $g(x)=\log f(x)$. If $g$ is concave and has a unique maximum $x^{*}$ on $D$, then $f$ has the same unique maximum $x^{*}$ on $D$.

Proposition 2 The function $f$ defined on $D=\left[1, \infty\left[{ }^{n}\right.\right.$ by $f\left(x_{1}, x_{2}, \ldots, x_{n}\right)=\sum_{l=1}^{k} \log (1-$ $\left.\prod_{j \in K_{l}} q_{j}^{x_{j}}\right)$ is concave.

For proofs of Lemma 1 and Proposition 2 we can refer to Souissi et al. (1994).

After logarithmic transformation, the problem to be solved can be written as follows:

$\begin{array}{ll}\text { Maximize } & \sum_{l=1}^{k} \ln \left[\left(1-\prod_{j \in K_{l}}\left(1-p_{j}\right)^{m_{j}}\right)\right] \\ \text { s.t. } & \sum_{j=1}^{N} g_{i j}\left(m_{j}\right) \leq b_{i} \quad i=1,2, \ldots, r \\ \text { and } & m_{j} \in \mathbb{I}^{*} \quad j=1, \ldots, N\end{array}$

By lemma 1, the objective function of (P2) has a unique maximum . Then the problem is to maximize a non-linear objective function having a unique maximum subject to linear or nonlinear convex constraints, with $m_{j}$ integers. To this end we propose the following algorithm:

\section{ALGORITM}

This algorithm is based on the branch and bound principle

1 - Relax the integrality constraints and solve the problem with the solver MINOS5 for nonlinear programming.

2 - IF the solution is integer THEN stop, the solution is optimal. ELSE, GO TO 3.

3 - Put the upper bound UB as the value of the objective function of the nonlinear program of the first step. 
Do a depth-first search to obtain an integer solution with cost $C_{0}$ which will constitute a lower bound LB on the objective function.

4 - Go up in the tree and explore the other branches updating lower and upper bounds.

We have developed a code of this algorithm in GAMS language and computed the optimal solution for different examples in the literature.

Recently we have purchased DICOPT, the MINLP solver, and we have written a GAMS code using this solver to test the same examples.

Example 1: The system considered in this example is the same as represented in Figure 0.1 and it was studied in Aggarwal (1976) and Shi (1987). The problem

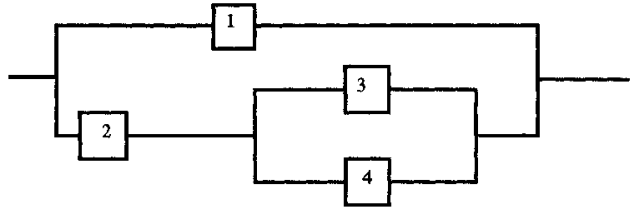

Figure 0.1: system $S$ studied by Shi and Aggarwal

is to maximize system reliability under budget and weight constraints. Component relibilities, costs and weights are given in Table 1 and the total budget allocated is 40 .

Table 1 Data of example 1.

\begin{tabular}{ccccc}
\hline component $\mathbf{i}$ & $\mathbf{1}$ & $\mathbf{2}$ & $\mathbf{3}$ & $\mathbf{4}$ \\
\hline reliability $p_{i}$ & 0.80 & 0.75 & 0.70 & 0.65 \\
costs $c_{i}$ & 6 & 4 & 3 & 2 \\
weights $w_{i}$ & 9 & 4 & 4 & 3 \\
\hline
\end{tabular}

The results of the different studies are drawn in Table 2 . We see that we have improved the results obtained by D.H. Shi (1986), which are better than those obtained by Aggarwal (1976).

Table 2 Comparison of computational results

\begin{tabular}{cccc}
\hline Method & K.K. Aggarwal & D.H. Shi & Our Algorithm \\
\hline Optimal solution & $1,3,2,3$ & $2,2,1,3$ & $3,1,1,1$ \\
Reliability value & 0.9961 & 0.9970 & 0.99737 \\
\hline
\end{tabular}

\section{Further examples and comparisons}

Recently we have got the DICOPT ++ solver for mixed integer nonlinear programming and we have tested many examples drawn from literature using our algorithm. We have then compared 
the results obtained by the code using branch and bound and those obtained by the code using the DICOPT ++ solver to those obtained by the authors of the different given examples. Some of the results of these computations are given in Table 3.

Table 3 Some computational results using the first lower bound.

\begin{tabular}{ccccc}
\hline author & known opt (1) & B. and B. (2) & DICOPT (2) & L. B. (3) \\
\hline Misra $(\mathrm{c}=70)$ & $2,2,1,2,1$ & $1,1,2,3,2$ & $3,2,2,1,1$ & \\
5 subs & 0.987370 & ${ }^{*} 0.990188$ & 0.990188 & 0.9900046 \\
Misra(c=90) & $4,3,1,2,1$ & $3,2,1,3,2$ & $3,2,1,3,2$ & \\
5 subsy & 0.998844 & ${ }^{*} 0.998963$ & 0.998963 & 0.9989553 \\
Misra(c=110) & $6,3,1,2,1$ & $4,2,2,4,2$ & $4,2,2,4,2$ & \\
5 subsy & 0.999776 & ${ }^{*} 0.999809$ & 0.999809 & 0.9998085 \\
Yasutake & $1,1,2,2,1$ & $1,1,2,2,1$ & $1,1,2,2,1$ & \\
10 subsy & $1,1,2,3,2$ & $1,1,2,3,2$ & $1,1,2,3,2$ & \\
& 0.983708 & 0.983708 & 0.983708 & 0.9819554 \\
Luuus & $3,4,6,4,3$ & $3,4,5,4,3$ & $3,4,6,4,3$ & \\
15 subsy & $2,4,5,4,2$ & $2,4,5,4,2$ & $2,4,5,4,2$ & \\
& $3,4,5,4,5$ & $3,4,6,4,5$ & $3,4,5,4,5$ & \\
& $0.945613^{*}$ & 0.944860 & $* 0.945613$ & 0.9448599 \\
\hline
\end{tabular}

\section{ALGORITHM BASED ON MINIMAL PATH SETS}

In this section we propose to use the bound of Prékopa to approximate the objective function of Problem (P1).We make also the assumption that the components are statisticaly independent.

\subsection{Prékopa's bounds}

Prékopa (1988) has presented a method, based on the so called Bool-Bonferroni inequalities, to obtain sharp lower and upper bounds for the probability that at least one-out-of a number of events in an arbitrary probability space will occur.

The knowledge of some of $S_{k}$ 's $k=1, \ldots, m$, allows us to obtain a lower bound for the probability $P[\mu \geq 1]$ by solving the following linear programming

$$
\begin{aligned}
& \text { Minimize } \sum_{i=1}^{n} v_{i} \\
& \text { s.t. } \quad \sum_{i=1}^{n}\left(\begin{array}{l}
k \\
i
\end{array}\right) v_{i}=S_{k} \quad k=1, \ldots, m, \\
& \text { and } \quad v_{i} \geq 0 \quad i=1, \ldots, n \text {, }
\end{aligned}
$$

Similarly, to obtain an upper bound for $P[\mu \geq 1]$, we maximize the objective function of the above linear program with the same constraints. In fact, the optimum value $V_{\max }$ of the 
objective function of the maximization problem can be greater than 1 . If this is the case, $V_{\max }>1$, then we know that the sharp upper bound is equal to 1 .

The optimum values $V_{\min }$ and $V_{\max }$ of problems mentioned above give sharp lower and upper bounds, respectively, of $P[\mu \geq 1]$

\section{Remark}

a) The more $S_{k}$ 's we know, the better are the bounds obtained by Prékopa's method.

b) To use Prékopa's algorithm for reliability problems, we have to know all minimal path sets and their probabilities, the probabilities of the intersection of all pairs of paths, and so on. These quantities are very difficult to determine.

\subsection{Sharp bounds for $P[\mu \geq 1]$ in case of $m=2$ and $m=3$.}

In this subsection we give the expressions of sharp lower and upper bounds on $P[\mu \geq 1]$ in term of $S_{i}$ 's for the case where $m=3$. These bounds have been provided by Boros and Prékopa (1989) where we can find other expressions of these bounds.

a - case $\mathbf{m}=2$ : the lower bound is given by: $P(\mu \geq 1) \geq \frac{2}{l+1} S_{1}-\frac{2}{l(l+1)} S_{2}$. This bound is sharp for $l-1=\left\lfloor\frac{2 S_{2}}{S_{1}}\right\rfloor$ where $\lfloor$.$\rfloor means the largest integer smaller than the number inside.$ This bound was obtained before by Dawson and Sankoff, Kwerel, Galambos.

b - case $\mathbf{m}=3:$ the lower bound is given by: $P(\mu \geq 1) \geq \frac{l+2 n-1}{(l+1) n} S_{1}-\frac{2(2 l+n-2)}{l(l+1) n} S_{2}+\frac{6}{l(l+1) n} S_{3}$. This bound is sharp for $l=1+\left\lfloor\frac{2\left((n-2) S_{2}-3 S_{3}\right)}{(n-1) S_{1}-2 S_{2}}\right\rfloor$

\section{APPLICATION TO RELIABILITY FIELD}

Let $S$ be a coherent system as defined by Balow and Prochan (1975) and designate by $P_{i}$ $(i=1,2, \ldots, p)$ the $i^{\text {th }}$ minimal path set of $S$. The events considered in this section are the minimal path sets occurence. Let $E_{r}$ be the event that all components in the minimal path set $P_{r}$ work. Then $P\left[E_{r}\right]=\prod_{i \in P_{r}} p_{i}$. The system functions if and only if at least one of the minimal path sets operates. Then the system operation corresponds to the event $\bigcup_{r=1}^{p} E_{r}$ (at least one path set is functioning), hence $P[S$ functions $]=P\left[\bigcup_{r=1}^{p} E_{r}\right]=P[\mu \geq 1]=R(S)$. The exact value of $R(S)$ is given by $P[\mu \geq 1]=\sum_{k=1}^{n}(-1)^{(k-1)} S_{k}$. Its computation necessitates the knowledge of all the $S_{k}$ 's which is a difficult, even impossible task to perform. Hence we propose to approach this probability by the bounds obtained by Prékopa and Boros (1989).

\subsection{Redundancy optimization using Prékopa and Boros bounds}

We propose to maximize the system reliability using redundancy. But, as we mentioned above, the problem is that we haven't the exact expression of the reliability of the system. We shall use Prékopa and Boros bounds instead.

Let $\alpha_{j}=-\ln \left(1-p_{j}\right)$ and $z_{j}=\ln \left(1-e^{-\alpha_{j} x_{j}}\right)$ and denote by $\mathcal{Z}_{j} j=1, \ldots, n$ the set 
$\left\{z_{j}=\ln \left(1-\exp \left(-\alpha_{j} x_{j}\right)\right), x_{j} \in \mathbb{N}\right\}$ and $\mathcal{Z}=\bigotimes_{j=1}^{n} \mathcal{Z}_{j}$

In the case of $\mathrm{m}=3$ the problem can be stated as follows:

Maximize $\frac{l+2 n-1}{(l+1) n} \sum_{i=1}^{p_{1}} \exp \left(\sum_{j \in P_{i}^{1}} z j\right)+\frac{6}{l(l+1) n} \sum_{i=1}^{p_{3}} \exp \left(\sum_{j \in P_{i}^{3}} z j\right)+$

$\frac{2(2 l+n-2)}{l(l+1) n} \sum_{i=1}^{p_{2}} \exp \left(\sum_{j \in P_{i}^{2}} z j\right)$

s.t. $\quad \sum_{j=1}^{n}-\beta_{j} \ln \left(1-e^{z_{j}}\right) \leq c$

and $z_{j} \in \mathcal{Z}_{j}$

where $P_{i}^{k}$ is a union of $k$ different minimal path sets ( $k$ different minimal path sets work simultaneously ).

\subsection{Convexity of the studied problems}

In this subsection we will show that the problems mentioned above are of the type of minimisation of a difference of two convex functions over a convex domaine.

Lemma 2 Let $f$ be a numerical concave funcion from $I \subseteq I R$ to $\mathbb{R}$ and let $\varphi$ be a concave function from $\mathbb{R}$ to $\mathbb{R}$ which is nondecreasing. Then $h(x)=(\varphi(f(x))$ is concave on $I$.

Lemma 3 Let $A$ be a linear transformation from $\mathbb{R}^{n}$ to $\mathbb{R}^{m}$. Then for each convex function $g$ on $\mathbb{R}^{m}$, the function $g A$ defined by $g A(x)=g(A(x))$ is convex on $\mathbb{R}^{n}$.

Proposition 3 The functions $S_{k}$ 's are convex and the domain $D$ of Problem (P5) is convex.

Proof: immediate from Lemma 2 and Lemma 3. The reader can refer to Souissi et al. (1995).

Using Tuy (1987) formulation of the minimisation of a difference of two convex functions, we can write the Problem (P5) into the equivalent form:

$$
\begin{aligned}
& \text { Maximize } t-\left[\frac{l+2 n-1}{(l+1) n} \sum_{i=1}^{p_{1}} \exp \left(\sum_{j \in P_{i}^{1}} z j\right)+\frac{6}{l(l+1) n} \sum_{i=1}^{p_{3}} \exp \left(\sum_{j \in P_{i}^{3}} z j\right)\right] \\
& \text { s.t. } \quad \frac{2(2 l+n-2)}{l(l+1) n} \sum_{i=1}^{p_{2}} \exp \left(\sum_{j \in P_{i}^{2}} z j\right) \leq t \\
& \quad \sum_{j=1}^{n}-\beta_{j} \ln \left(1-e^{z_{j}}\right) \leq c \\
& \text { and } \quad z_{j} \in \mathcal{Z}_{j}
\end{aligned}
$$

The objective function of (P6) is now a concave function. If we relax the condition that $z_{j}$ is discrete to that $z_{j}$ is continuous, $z_{j} \in\left[\ln \left(1-e^{-\alpha_{j}}\right), \ln \left(1-e^{-10 \alpha_{j}}\right)\right]$, then the constraint set $D$ (called domain) is a closed bounded and convex. 


\section{CONCLUSION}

Using our approach we have, in most examples of literature, improved the known solutions. For example, the reliability of the bridge system, have been improved by comparison to Misra results, respectively for $c=70, c=90$ and $c=110$. We have also improved the result of Shi (1986) for the system with 2 constraints. However for the Luus system we note that the solution obtained by the code of branch and bound is worse than the solution obtained by Luus, but we obtain the same optimal solution as Luus when using the DICOPT++ solver.

The computation of the absolute and relative errors shows that the lower bounds obtained with the minimal cuts in section 2 and with the minimal paths in section 3 are very tight to the exact system reliability values. These errors are comprised between $5 * 10^{-7}$ and $3.0377 * 10^{-3}$.

\section{REFERENCES}

Aggarwal, K.K. (1976) Redundancy optimization in general systems. IEEE Transactions on Reliability, R-25 (5), pp 330-2.

Ball, M.O. (1980) Complexity of Network Reliability Computations. Networks, 10, $153-65$.

Barlow, R.E. and Prochan, F. (1975) Statistical theory of reliability and life testing: probability models. Holt, Rinehart and Winston, New York.

Bellman, R. and Dreyfus, S. (1958) Dynamic programming and the reliability of multicomponent devices. Oper. Res., Vol 6, pp 200-6.

Boros, E. and Prékopa, A. (1989) Closed form tow-sided bounds for probabilities that at least $r$ and exactly $r$ out-of $n$ events occur. Mathematics of Operations Research, 14, 317-42.

Luus, R. (1975) Optimization of system reliability by a new nonlinear integer programming procedure. IEEE Transactions on Reliability, R-24(1), 14-16.

Misra, R.B. and Agnihotri, G. (1979) Pecularities in optimal redundancy for a bridge network. IEEE Transactions on Reliability, R-28(1), pp 70-2.

Prékopa, A. (1988) Boole-Bonferroni Inequalities and Linear Programming. Operations Research, Vol 36, pp 145-62.

Prékopa, A. (1990) Sharp Bounds on Probabilies Using Linear Programming. Operations Research, Vol 38, pp 227-39.

Shi, D.H. (1987) A New Heuristic Algorithm for Constrained Redundancy-Optimization in Complex Systems. IEEE Transactions on Reliability, R-36(5), 621-3.

Souissi, M. and Smeers, Y. (1994) Constrained redundancy optimization of coherent systems. Worksop On Reliability and Maintenance Modeling., Amsterdam.

Souissi, M. and Smeers, Y. (1995) Redundancy optimization of coherent systems. Nantes Eurodays on Industrial Systems Design., Nantes, France.

Tillman, F.A. Hwang, C.L. and Kuo, W. (1977) Optimization techniques for system reliability with redundancy - a review. IEEE Transactions on Reliability, R-26(3), pp 148-55.

Tuy, H. (1987) Global Minimization of a Difference of two Convex Functions. Mathematical Programming Study, pp. 150-82, 